\title{
Electron Holography for Everyone - Here It Comes
}

\section{Edgar Voelkl}

HoloWerk LLC, 4 Seneca Forest Ct, Germantown, MD 20876

\section{HoloWerk@mac.com}

For seventy years electron holography has been attempted with varying degrees of success. I believe the time is right to produce an electron holography microscope that would make electron holography a mainstream analysis tool.

Background. Since Zernike's invention in 1933, imaging the phase of the complex object wave has been an ongoing effort [1]. Of the many different approaches, (in-line) holography, invented by Gabor in 1948, is the most famous [2]. The invention of the electron biprism by G. Möllenstedt in 1954 and the long-time classified work by E.N. Leith released in 1962 opened the door to off-axis electron holography (EH), the standard EH technique today [3,4]. Further methods like Scherzer focus, defocus series, and differential phase contrast highlight the importance of imaging the phase, which could be summarized by simply stating that the sample first and foremost causes a change in the phase of the electron wave [5-7]. Of the phase imaging techniques, Zernike-type phase contrast and specifically differential phase contrast have seen a recent transition to mainstream popularity because of their "ease of use" through careful hardware and software/workflow development.

Recent electron holography developments. Off-axis EH, however, remains a complex application largely because of the physics involved: (a) amplitude splitters for large angles do not exist for electrons (requiring the area of interest to be near a sample-free area); (b) electrons are fermions, causing a significant limitation for lateral coherence; and (c) the hologram must be processed first before the phase image becomes visible.

Nonetheless, at Oak Ridge National Laboratory, early work on extending phase sensitivity via averaging stroboscopically acquired holograms started in 1995 [8]. However, the stability of the illumination system denied that goal back then. In 2005, the arrival of Cs correctors brought about the needed stability of the illumination system. In 2007 an exposure time of several minutes with minimal loss of fringe contrast was announced [9]. The increased illumination stability also allowed for the averaging of stroboscopically acquired holograms reported in 2010 [10], delivering high sensitivity, for example, to small magnetic fields. Related workflow development was announced in 2018 following recent computer-based improvements [11].

Single-electron detectors. Around 2005 a key improvement on the detector side arrived quietly: single-electron detection. Conventional cameras negatively impact phase resolution in $\mathrm{EH}$ because fine interference fringes must be recorded at low sampling rates. Single-electron detectors on the other hand have very good specifications for the modulation transfer function (MTF) and the detection quantum efficiency (DQE), which make them ideal for EH. As shown in 2015, a hologram recorded on a single-electron detection camera vastly improves the information contained in the phase image (conventionally referred to as phase resolution). With an average electron count of four per pixel, the direct image showed neither the sample nor the interference fringes-only noise. After processing, comparatively welldefined phase images were obtained that allowed the sample thickness to be measured to within $\sim 2 \mathrm{~nm}$ [12].

Thin samples, and definitely electric and magnetic fields, shift the phase of the electron beam, and so it comes as no surprise that the phase image can carry a strong signal. In the classical image of electrons as particles, half of them go through the sample and the other half provides the reference beam. In the quantum mechanical description, however, each electron "interacts" with the sample, contrary to the classical particle perspective (ignoring inelastically scattered electrons). Thus $\mathrm{EH}$ holography not only records the strongest signals from the sample (it records amplitude and phase), it also benefits from electrons that classically would not be considered as interacting with the sample at all. This makes EH the most sensitive phase imaging technique.

Not yet a mainstream technique. Even with sophisticated workflows, EH is not a mainstream application for one simple reason: changing magnification interrupts continuous imaging of the phase. There are two aspects of this: (a) the magnification change typically includes removing the sample from the field of view, re-aligning the illumination

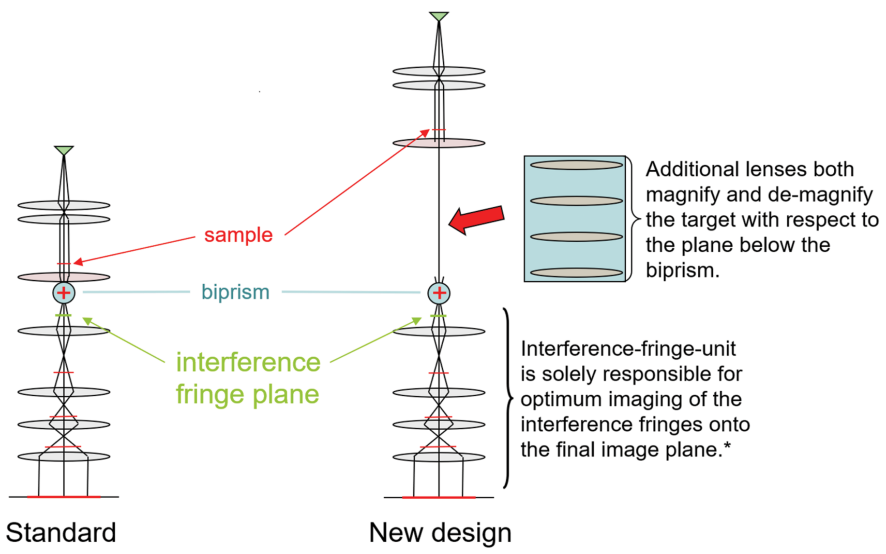

Figure 1: Adding electron lenses and deflectors (not shown) between the objective lens and the biprism (blue circle) of a standard transmission electron microscope would allow electron holography to become a mainstream application, as explained in the text. 
and the imaging lenses, finding the sample, re-focusing, and correcting beam tilt; and (b) the change of object magnification also changes the magnification of the interference, requiring the adjustment of the voltage at the biprism and at least one of the imaging lenses. Both (a) and (b) have to be balanced iteratively, adding to the long delays when changing magnification. Thus changing from an overview of the specimen area to a more detailed view rudely interrupts live phase imaging-and that is where most users lose interest in the technique.

The solution. The solution is to separate the magnification of the interference fringes (with respect to the camera) from the magnification of the object. An approach to this was proposed in 2001 [13]. As shown in Figure 1, adding lenses between the objective lens and the biprism allows magnification changes of the sample while the original lenses below the biprism maintain the interference fringes with respect to the camera. Adding lenses to the optical train in the suggested area should not be complicated; Cs correctors are more complex, and we know how to handle them.

I believe it is time for this simple modification to be made in a well-designed holography microscope. Such a microscope could be used like any other transmission electron microscope, but it would allow imaging the object simultaneously in its intensity and phase, live in real time. Then magnetic and electric fields become visible supporting investigations of $\mathrm{p}-\mathrm{n}$ junctions, magnetoresistive RAM, and electric fields around toner particles. At high resolution, local crystal tilt becomes obvious on the unit-cell scale, and biological objects would show much improved contrast even under low dose conditions - to name a few applications. Thus, electron holography could truly evolve into a mainstream technology and provide in real time all the information the electron wave contains.

\section{References}

[1] "Frits Zernike," Wikipedia, The Free Encyclopedia, accessed June 3, 2018.

[2] D Gabor, Nature 161(4098) (1948) 777-78.

[3] G Möllenstedt and H Düker, Naturwissenschaften 42 (1955) 41.

[4] EN Leith and J Upatnieks, J Opt Soc Am 52(10) (1962) 1123-30.

[5] O Scherzer, J Appl Phys 20 (1949) 20.

[6] HW Zandbergen and DV Dyck, Microsc Res Techniq 49(3) (2000) 301-23.

[7] H Rose, Optik 39 (1973-74) 416.

[8] E Voelkl et al., Scanning Microscopy 11 (1997) 407-16.

[9] D Cooper et al., Appl Phys Lett 91 (2007) 143501.

[10] E Voelkl and D Tang, Ultramicroscopy 110 (2010) 447-59.

[11] E Voelkl and A Ponce Microsc Microanal 24 (Suppl 1) (2018) 1462-63.

[12] E Voelkl et al., Microsc Microanal 21 (Suppl 3) (2015) 1951-52.

[13] E Voelkl et al., Electron holography microscope. US Patent 6,617,580, filed December 27, 2001.

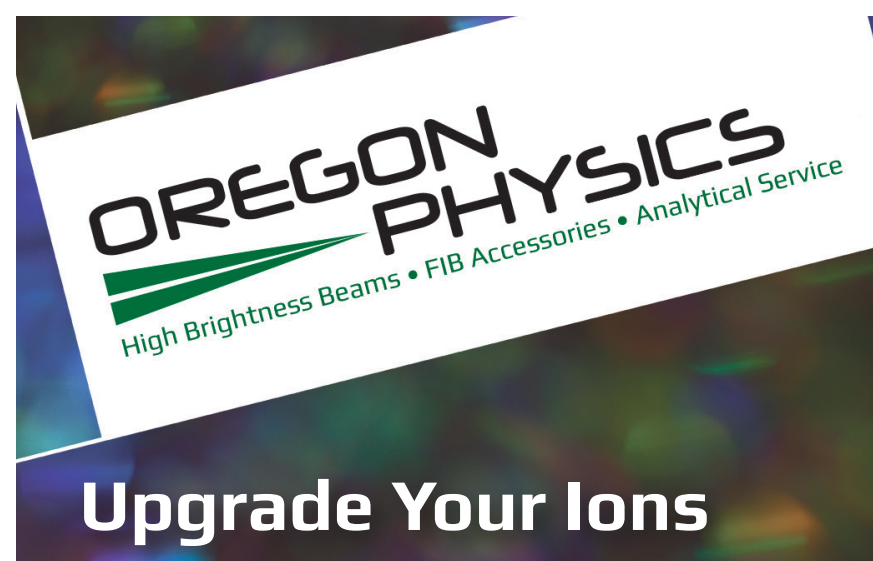

Hyperion $^{\text {TM }}$ Dual Polarity Ion Sources are now available as direct upgrades from Oregon Physics for FEI FIB 200, PHI Adept 1010, and Cameca NanoSIMS, IMS F series, and 12XX series instruments.

\section{Upgrade your ion source to benefit from:}

- Longer source lifetime

- Better image resolution

- Improved depth profiling (SIMS)

\section{- Higher currents for milling (FIB)}

Oregon Physics' Hyperion ion sources are designed to bolt-on to your existing optical system for easy implementation.

How will Hyperion improve your research? Learn more at Oregon-Physics.com or call us to discuss your requirements.

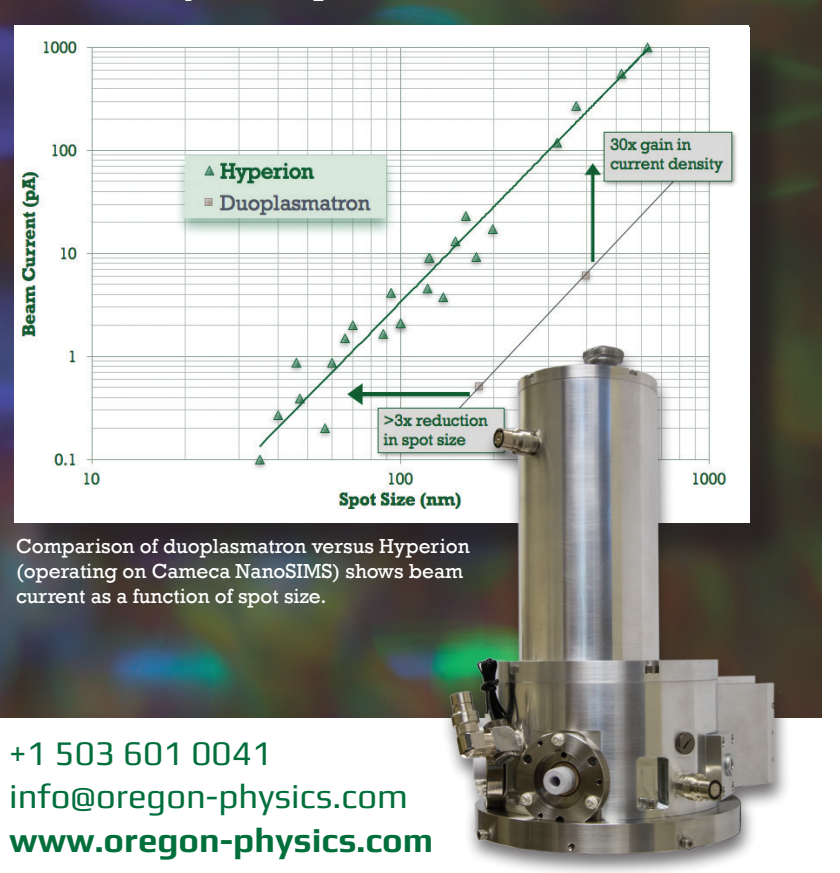

